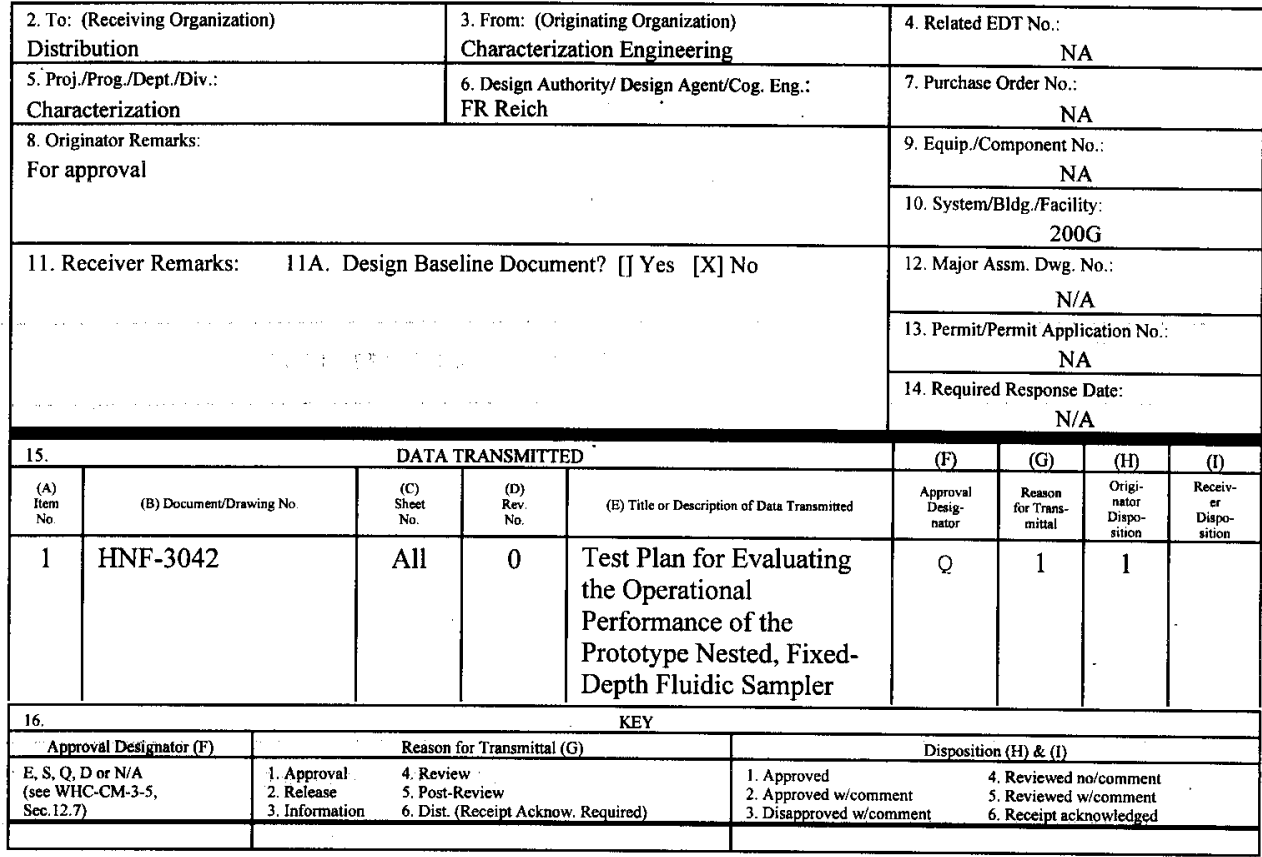

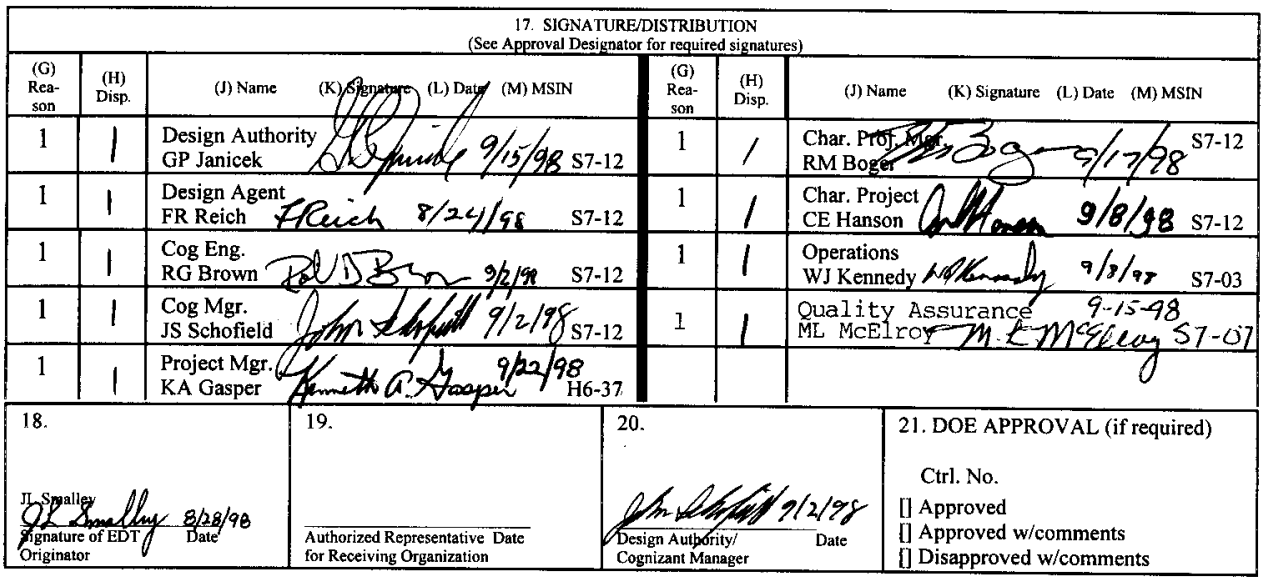

BD-7400-172-2 (05/96) GEF097 


\title{
TEST PLAN FOR EVALUATING THE OPERATIONAL PERFORMANCE OF THE PROTOTYPE NESTED, FIXED-DEPTH FLUIDIC SAMPLER
}

\author{
FR Reich \\ JL Smalley \\ COGEMA Engineering Corporation, Richland, WA 99352 \\ U.S. Department of Energy Contract DE-AC06-96RL13200
}

EDT/ECN: 622083

Org Code: $08 \mathrm{E} 00$

B\&R Code: EW3120074
UC: 2070

Charge Code: W7P55

Total Pages: 45

Key Words: $\quad$ waste sampling, double-shell, LAW, sampling system, at-tank analysis

Abstract:

The PHMC will provide Low Activity Wastes (LAW) tank wastes for final treatment by a privatization contractor from two double-shell feed tanks, 241-AP-102 and 241-AP-104. Concerns about the inability of the baseline "grab" sampling to provide large volume samples within time constraints has led to the development of a nested, fixed-depth sampling system. This sampling system will provide large volume, representative samples without the environmental, radiation exposure, and sample volume impacts of the current base-line "grab" sampling method. A plan has been developed for the cold testing of this nested, fixed-depth sampling system with simulant materials. The sampling system will fill the $500-\mathrm{ml}$ bottles and provide inner packaging to interface with the Hanford Sites cask shipping systems (PAS-1 and/or "safe-send"). The sampling system will provide a waste stream that will be used for on-line, real-time measurements with an at-tank analysis system. The cold tests evaluate the performance and ability to provide samples that are representative of the tanks' content within a 95 percent confidence interval, to sample while mixing pumps are operating, to provide large sample volumes (1-15 liters) within a short time interval, to sample supernatant wastes with over $25 \mathrm{wt} \%$ solids content, to recover from precipitation- and settling-based plugging, and the potential to operate over the 20 -year expected time span of the privatization contract.

TRADEMARK DISCLAIMER. Reference herein to any specific commercial product, process, or service by trade name, trademark, manufacturer, or otherwise, does not necessarily constitute or imply its endorsement, recommendation, or favoring by the United States Government or any agency thereof or its contractors or subcontractors.

Printed in the United States of America. To obtain copies of this document, contact: Document Control Services, P.O. Box 950, Mailstop H6-08, Richland WA 99352, Phone (509) 372-2420; Fax (509) 376-4989.

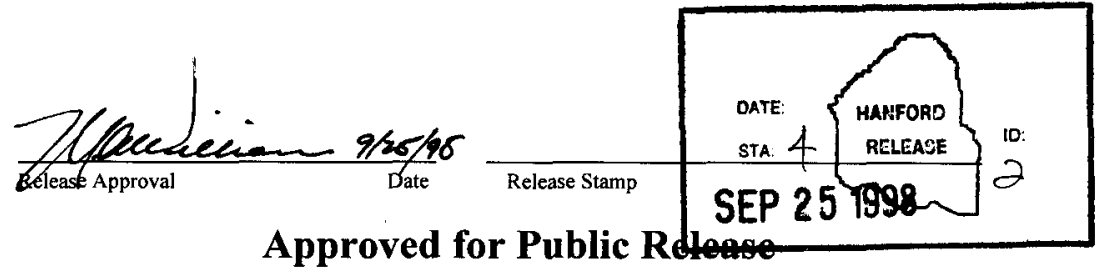


HNF-3042 REVISION 0

TEST PLAN FOR EVALUATING THE OPERATIONAL PERFORMANCE OF THE PROTOTYPE NESTED, FIXED-DEPTH FLUIDIC SAMPLER

Prepared for Characterization Engineering by

F. R. REICH

J. L. SMALLEY

COGEMA Engineering Corporation

August 1998 
TABLE OF CONTENTS

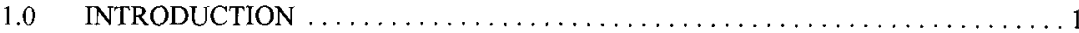

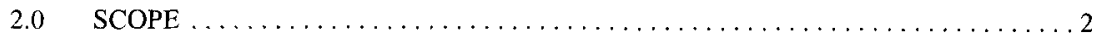

2.1 CONCEPTUAL NESTED, FIXED-DEPTH SAMPLING SYSTEM TESTS . . 2

2.2 PROTOTYPE NESTED, FIXED-DEPTH SAMPLING SYSTEM TESTS . . . . 2

3.0 PURPOSE AND OBJECTIVES $\ldots \ldots \ldots \ldots \ldots \ldots \ldots \ldots \ldots \ldots \ldots \ldots \ldots$

4.0 TEST BACKGROUND AND GUIDANCE $\ldots \ldots \ldots \ldots \ldots \ldots \ldots \ldots \ldots \ldots \ldots$

4.1 TEST SITE DESCRIPTIONS $\ldots \ldots \ldots \ldots \ldots \ldots \ldots \ldots \ldots \ldots \ldots \ldots$

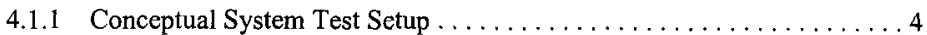

4.1 .2 Prototype System Test Site $\ldots \ldots \ldots \ldots \ldots \ldots \ldots \ldots \ldots \ldots$

4.2 ANALYSIS AND MEASUREMENT METHODS/TECHNIQUES $\ldots \ldots \ldots 5$

4.3 VALIDATION OF TEST SIMULANT PROPERTIES $\ldots \ldots \ldots \ldots \ldots \ldots \ldots$

4.4 REFERENCE MATERIAL SAMPLING $\ldots \ldots \ldots \ldots \ldots \ldots \ldots \ldots \ldots \ldots$

4.5 MEASUREMENT STATISTICS AND ACCURACY/PRECISION $\ldots \ldots \ldots .9$

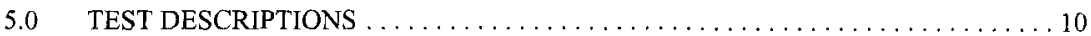

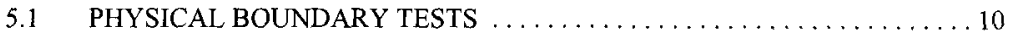

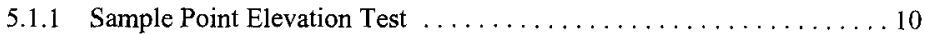

5.1 .2 Waste Depth Test ............................ 12

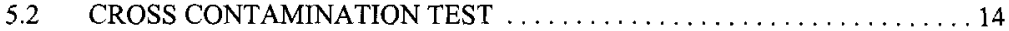

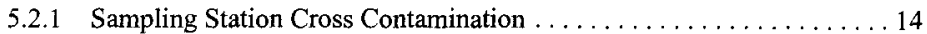

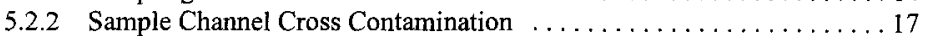

5.3 WASTE PHYSICAL PROPERTIES . . . . . . . . . . . . . . . 19

5.4 STARTUP AND OPERATION FROM A PLUGGED CONDITION $\ldots \ldots \ldots 21$

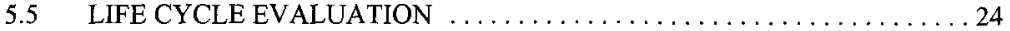

5.6 SAMPLING SYSTEM IN-TANK ORIENTATION $\ldots \ldots \ldots \ldots \ldots \ldots \ldots \ldots$

5.7 IN-TANK DEPLOYMENT AND OPERATION TESTING $\ldots \ldots \ldots \ldots \ldots 27$

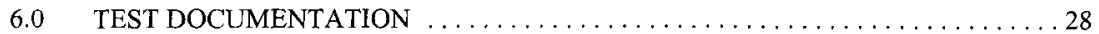

7.0 ORGANIZATIONAL RESPONSIBILITIES $\ldots \ldots \ldots \ldots \ldots \ldots \ldots \ldots \ldots \ldots \ldots$

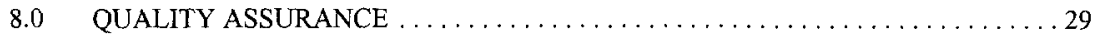

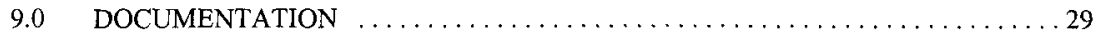

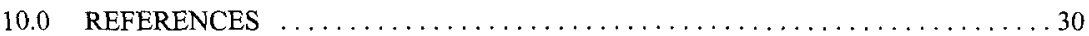




\section{LIST OF TABLES}

Table 1. Particle Size Range in Phase 1A Privatization Contracts ................. 11

Table 2-a. Simulant Compositions for Cross-Contamination Testing with the Conceptual

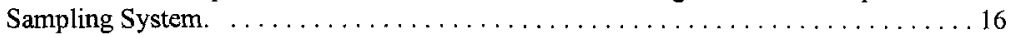

Table 2-b. Simulant Compositions for Cross-Contamination Testing with the Prototype

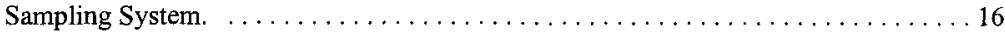

Table 3. Simulants for Evaluating Recovery From Plugged Conditions $\ldots \ldots \ldots \ldots \ldots 22$

\section{LIST OF FIGURES}

Figure 1. Single Fluidic Sampling Channel with Bottle Sampling Station Shown Deployed in a

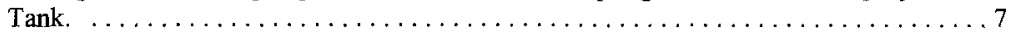

Figure A-1. Schematic Showing the Main Elements of a Fluidic RFD Based Pump. ....... 3

Figure A-2. Three Phases of Pump Operation; Top to Bottom, Refill, Drive, and Vent ...... 4

Figure A-3. Reverse Flow Diverter, RFD, Element ....................... 5

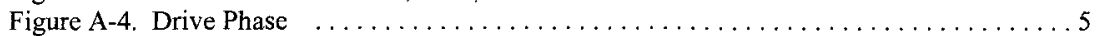

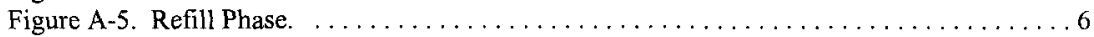

Figure A-6. Jet Pump Pair Used to Produce Drives for the Three Modes of Operation for the

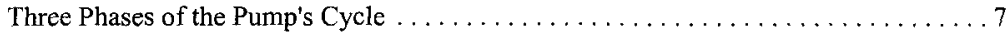

Figure A.7. Cycle Control with Timer and Pressure Control Systems . . . . . . . . . . 10

Figure A.8. Cycle Control with Timer and Pressure Control Systems. . . . . . . . . . . 11

\section{APPENDIX}

\section{APPENDIX A - FLUIDIC BASED SAMPLING SYSTEM COMPONENT DESCRIPTION}


HNF-3042, Revision 0

\section{TEST PLAN FOR EVALUATING THE OPERATIONAL PERFORMANCE OF THE PROTOTYPE NESTED, FIXED-DEPTH FLUIDIC SAMPLER}

\subsection{INTRODUCTION}

The final treatment of Hanford's Low Activity Waste (LAW) tank wastes will be completed through a privatization contract. The Project Hanford Management Contract (PHMC) will supply supernatant waste materials from two Hanford Site double-shell staging/feed tanks. Currently 241-AP-102 and 241-AP-104 are planned to be used as the staging tanks. Prior to transfer to the Privatization Contractor, the waste must conform to the envelope $A, B$, and C specifications (DOE 1997a and DOE 1997b), being negotiated in the TWRS Privatization Contract with BNFL, Inc. These feed envelopes are currently targeted to be dilute slurry/supernatant solutions with a maximum of five percent by volume solids, which is expected to change to two percent solids by weight.

The PHMC Team has identified four critical risks that may result in the PHMC Team paying for idle facility time (HNF-2019, Rev, 0, 1998):

- Facility Processing Rates: Privatization Contractor may expect a higher rate of feed than the PHMC Team can initially deliver.

- Waste Feed is Out of Specification: Waste feed may not meet Phase 1 specifications and will require unplanned adjustments.

- Waste Certification Strategy Not Yet Defined: Analytical results may not be available in time to meet schedules."

- Waste Feed Specification Disputes: Waste feed, which the PHMC Team analysis shows as being in specification, may be unacceptable to the private contractors based on the results of their analyses, thereby delaying waste feed delivery to the privatization contractors."

The current approved (baseline) for sampling tank liquids is "grab" sampling, which utilizes the "bottle on a string" technique. A major concern with this sampling method is that the length of time required to assure that a tank is well mixed and to extract representative samples may conflict with the schedule needs for staging batches of waste feed. Other concerns include a non-representative sampling because of a sampling bias with materials from upper tank elevations, a manual operation requiring operator exposure to radioactive wastes, and the potential impact of the laboratory time to complete the tank waste analysis.

In order to address these concerns, a nested, fixed-depth fluidic sampling system is being developed by the AEA Technology Engineering Services Corporation (AEA) for the U.S. 
developed by the AEA Technology Engineering Services Corporation (AEA) for the U.S. Department of Energy (DOE). The tasks to complete the nested, fixed-depth fluidic sampling system are in an Engineering Task Plan (Reich and Smalley 1998). This sampling system will quickly provide representative waste tank samples that can be used for laboratory analysis and/or be used to support an at-tank analysis system.

\subsection{SCOPE}

This test plan provides the guidance for the proof-of-principle testing of the conceptual nested, fixed-depth sampling system being developed by AEA and guidance for the testing of the future prototype sampling system at Hanford.

This test plan document does not address the at-tank analysis system or its interface with the nested, fixed-depth sampling system. Tests, measurements, and simulant materials are identified that will evaluate the operation and performance of the nested, fixed-depth sampling systems.

\subsection{CONCEPTUAL NESTED, FIXED-DEPTH SAMPLING SYSTEM TESTS}

The testing with the conceptual sampling system will be completed by AEA at their North Carolina facility. This system is not a full-scale system but, as indicated below, is a two-sample channel sampler with a limited valve and bottle sampling station. The tests that will be completed with this conceptual system are the following:

1. Physical Boundary Test (Section 5.1)

Sample Point Elevation Test (Section 5.1.1) - One test at approximately $20 \mathrm{ft}$ of elevation and one test at approximately $60 \mathrm{ft}$ of elevation.

2. Cross-Contamination Test (Section 5.2)

Sample Channel Cross-contamination ( Section 5.2.2)

3. Startup and Operation from a Plugged Condition (Section 5.4)

Precipitation Plugging (Section 5.4)

\subsection{PROTOTYPE NESTED, FIXED-DEPTH SAMPLING SYSTEM TESTS}

The prototype testing will be completed at Hanford with the full-scale, full-length prototype sampling system. Any test not addressed by the conceptual system will be completed with the prototype system. However, at this time it is not known if the tests completed with the conceptual system will be repeated. Archived data and system analysis may be sufficient for the Life-cycle Testing and In-tank Deployment and Operation Testing. 
HNF-3042, Revision 0

\subsection{PURPOSE AND OBJECTIVES}

The purpose of these tests is to evaluate and demonstrate the proof-of-principle of the nested, fixed-depth sampling systems that are being developed for deployment in the PHMC Team's $241-\mathrm{AP}-102$ and $241-\mathrm{AP}-104$ waste staging tanks. The object of the testing is to:

- demonstrate that the sampling system can extract waste material that is representative of the material at the sampling system inlets. This testing will evaluate the sampling performance with simulants that represent chemical and physical properties of the supernatant tank waste material anticipated in the Phase 1B privatization contract. The tests address waste materials, waste levels, and tank dimensional impacts. Note: this testing will not address the radiological aspects of the proposed sampling technique.

- identify and verify the potential impacts of other in-tank systems, such as the mixer pump.

- show that the nested, fixed-depth sampling system can provide representative, large waste samples within a time frame that supports the waste volume needs of the Privatization Contract.

The waste sampling objectives to support the Privatization Contract can be refined as follows:

- extract waste samples that are representative of the waste material in the PHMC Team's feed source and/or staging tanks (it is desired that these samples be representative of a tanks content within a 95 percent confidence interval)

- obtain representative waste samples from full and partially full tanks

- quickly providing relatively large volumes (two to 10 liters) of sample waste material, with minimal impact from weather conditions currently affecting the base-line, grab sampling method

- sample while other in-tank hardware is operating, including mixer pumps, decanting operations, etc., without impact to the sampling performance

- allow safe deployment in a tank riser and meeting tank interface (dimensions, loading, etc.), environmental, safety, and regulatory criteria

- have an expected operational longevity that approaches the anticipated 20-year time span of the privatization contract use the current $500-\mathrm{ml}$ glass sampling bottle as the sampling container and interface with the Site's cask shipping systems (PAS-1 and/or "safe-send"TM cask systems).

\subsection{TEST BACKGROUND AND GUIDANCE}

Appendix A provides a description of the basic principles and components for the fluidic-based sampling system. 
HNF-3042, Revision 0

\subsection{TEST SITE DESCRIPTIONS}

The conceptual nested, fixed-depth sampling system will be tested at the AEA Technology's Charlotte, North Carolina, facility. The test hardware will be set up in the existing high bay workshop area adjoining the AEA Technology offices, where previous testing of fluidic devices for deployment on DOE sites has been successfully conducted.

The prototype nested, fixed-depth sampling system will be tested at the Hanford site. A cold test facility will be set up that can support the testing of a full-scale/length sampling system. This test site will be set up prior to the fabrication and delivery of the prototype sampling system.

\subsubsection{Conceptual System Test Setup at AEA Facilities}

An example of a single sampling channel for a nested, fixed-depth sampling system with reverse flow diverter (RFD), charge vessel, bottle sampling station, and jet pump is shown in Figure 1. Additional details on the sampling system components and operation are included in Appendix A. Figure 1 shows the sampling channel as it might be installed in a large waste tank.

The test setup that AEA will use to test the conceptual system will consist of a single sampling channel (Figure 1). The vacuum and air pressure needed to cycle this pumping system will be supplied by the jet pump system shown above the charge vessel. The charge vessel fills with waste through the RFD using a vacuum. After the fill operation is completed, the charge vessel is pressurized, which forces the waste through the RFD and up to the bottle sampling station. The waste movement past the sampling needle draws a partial vacuum in the sample bottle. After the pressure is removed from the charge vessel, the waste will run back into the tank. However, the vacuum in the bottle will draw waste into the bottle through the needle. The waste that moves past the bottle station is shown being returned to the tank. Additional details on the operation of this fluidic system are included in Appendix A.

The fluidic pump will be comprised of a cylindrical, carbon steel (or stainless steel) charge vessel approximately 64" $\mathrm{4}$ " internal diameter, with a 1" diameter RFD pumping element connected to the base by bolted flanged connections. The charge vessel will be connected via flexible hoses and a manually operated ball valve to a primary controller. The fluidic pump will be mounted in a supply tank (simulant tanks) of approximately 50-gallon capacity (this will ensure that sampling does not deplete tank volume by more than 5-10 percent).

The primary controller will consist of a single jet pump pair operating on a compressed air supply provided by a portable diesel powered compressor. Air flow to the jet pump pair will be controlled by the secondary controller or electronic pressure control unit. 
Each RFD will be connected, via flexible hoses and manually operated ball valve, to a single sample tee. Sampling will take place at the sample tee by means of a vertical sample needle. Needles of differing length or diameter may be attached to the sample tee. When sampling is to take place, the sample bottle, fitted with a suitable lid and seal, will be placed over the needle.

The sample tee will be fixed to a scaffold tower, approximately $60 \mathrm{ft}$. in height, which will provide access to the tee to carry out and observe sampling operations (the actual "lift" distance in AP-104 and -102 is in the 55-57 ft range, which will be the dimensions for the prototype system and the tests performed with it). It will also be possible to simulate different vertical separations between tee and sample point by raising or lowering the position of the tee on the scaffold tower. The sample tee and flexible hoses will all be constructed from transparent polymer material allowing the operation of the sampler to be clearly observed. Both samplers and feed tanks will be located at the base of the scaffold tower. Primary and secondary controllers will be securely mounted adjacent to the samplers.

AEA Technology will employ an independent testing contractor to carry out tests on samples during the test program. Details of the measurement systems to be employed in the test program will be confirmed after appointment of the testing contractor. The measurement techniques in Section 4.2 will be used in analyzing test samples taken. Accuracy and error bands attributable to the sample analysis procedures employed will be described in the final report.

\subsubsection{Prototype System Test Site}

Details of the test site for the cold testing of the full-scale/length prototype sampling system at Hanford have not been developed. Work in FY 1999 will identify the location and features of this test facility. The test facility will be designed to support the completion of all the testing, including needed life-time and operational performance tests described below.

\subsection{ANALYSIS AND MEASUREMENT METHODS/TECHNIQUES}

The analysis of grab samples and samples extracted with the conceptual nested, fixed-depth sampling system at AEA's facilities will be completed using the following:

Particle size distribution - This will be measured by microscopic examination as per ASTM F312.

Solids wt\% content - This will be measured by filtration and evaporation at $105^{\circ} \mathrm{C}$ per EPA 160.3. 
HNF-3042, Revision 0

Viscosity -

Viscosity will be measured using rotating bobbin and cup apparatus. Contraves, Haake, or Brookfield apparatus may be used dependent upon shear range.

Similar measurement methods are planned to be used with the prototype system at the Hanford cold test site. 
HNF-3042, Revision 0

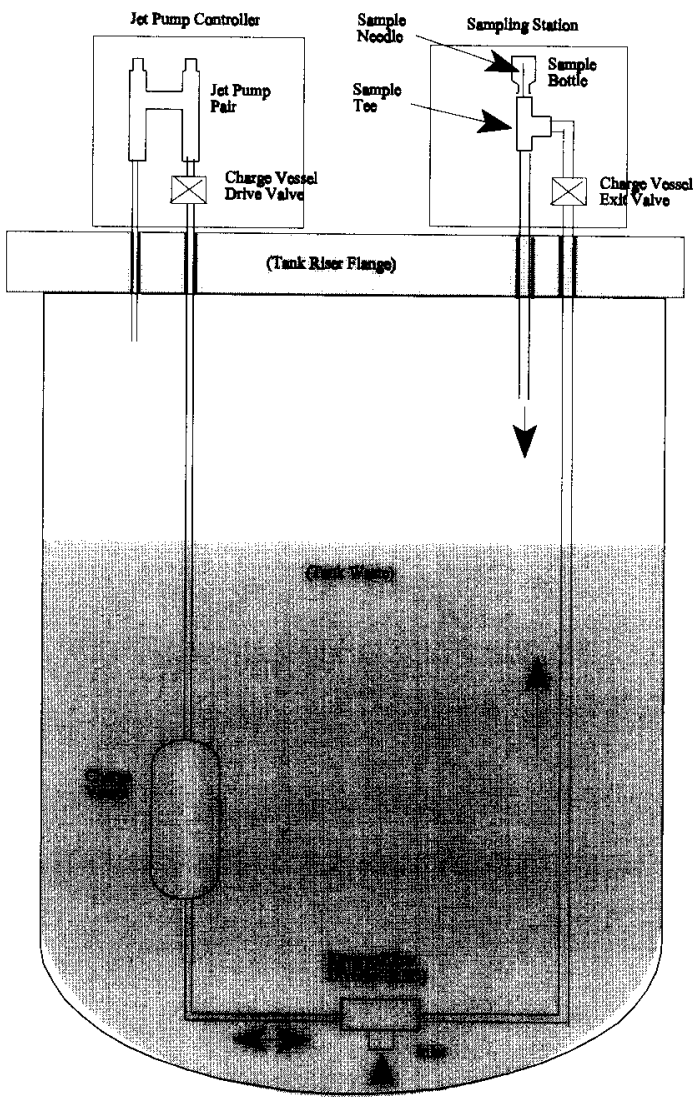

Figure 1. Single Fluidic Sampling Channel with Bottle Sampling Station Shown Deployed in a Tank. 
HNF-3042, Revision 0

\subsection{VALIDATION OF TEST SIMULANT PROPERTIES}

The properties of the simulants will be measured (characterized) and validated for each of the system tests using two independent methods. Initially, accurate measurements (to 0.1 percent) of the simulant's constituents (volume or weight of each constituent, particulate distributions, etc.) will be recorded during the makeup of a simulant. During each test, reference simulant material samples will be taken using grab sampling and analyzed, along with samples obtained by the nested, fixed sampling system (wt\% solids, particulate distribution, shear strength, etc.). The grab samples will be considered the baseline or reference for assessing the performance of the sampling system. These reference samples will be taken very close to the inlets of the prototype test hardware and at the same time that the "test performance indicating" samples (bottle samples taken by the sampling system) are taken. This procedure will minimize the potential for interfering errors from other non-sampling system sources (e.g., lack of homogenous mixing in the vessels holding the simulants).

The tests described in this document identify particulate size distribution and wt $\%$ solids as the two main parameters for measuring the performance of the sampling system. Other physical property measurements may be necessary to ensure that a simulant meets specifications. The data recorded during simulant makeup will be used to assess the potential presence and impact of other, non-sampling system, error sources (non-sampling system error sources, such as incomplete mixing in the simulant vessels). The simulant makeup and the reference sample data sets will provide validation data that the test simulant properties are in conformance with the simulant specification (Rinker 1998a).

\subsection{REFERENCE MATERIAL SAMPLING}

References samples, when required, will be obtained using a grab sampling process. The ASTM standard, Standard Practice for Sampling Industrial Chemicals (ASTM E300-92), provides guidance for this grab sampling, which is the current Hanford baseline method for obtaining liquid and slurry tank waste samples. Care and diligence should be used in the acquisition of the grab samples. Section 10.0 of the ASTM standard covers the sampling of "Simple Liquids" while Section 35.0 covers "Slurry Sampling." The practices, procedures, limitations and cautions, etc., described in these sections should be used as guidance in the planning and execution of grab sampling. The grab sampling should use a 100-500-ml size sample bottle/flask with other recommendations and physical characteristics as described in ASTM E300-92 (mouth size, total sample volume, etc.). 
HNF-3042, Revision 0

\subsection{MEASUREMENT STATISTICS AND ACCURACY/PRECISION}

The performance objective of the sampling system is to provide samples that have chemical and physical properties that are within $+/-5.0 \%$ of the tank waste with a 95 percent confidence level. The sampled waste reference will be the material directly adjacent to a sampling channel inlet (this reference material will be obtained by careful grab sampling). In tests where the quality of material is the test issue (such as in the 5.1 Physical Boundary Tests, 5.2 Cross-contamination Test, and 5.3 Physical Property Tests, discussed below) it is recommend that five to seven test repetitions be completed. For tests where performance and not material quality is the issue (Sections 5.4, 5.5, 5.6, and 5.7), repetitions of two to three should be considered. However, a single, carefully executed sample/test may be sufficient. For example, if care is used in setting up and characterizing plugging conditions and making recovery-based measurements, a single test may suffice.

The following definitions will apply in this testing work:

- Sensitivity: This is the relationship between an instrument's output and the parameter it is responding to. It is used to specify the minimum detectable change in a parameter that the instrument will respond to (most often this is the signal-tonoise ratio of the instrument).

- Accuracy: This is an indication of how close a measured value (or a group of measured values) is to the "true" value or an accepted standard. How close together the group of measurements are with respect to each other is not an issue. With a group of repeated measurements, there is high accuracy if the mean value of the measurement group is very close to the "true" value. However, there can be a significant span (see definition below) between the individual measurement values.

- Precision: This is an indication of how repeatable a measurement is (the spread of the grouping of repeated measurements). Precision is different from accuracy in that it is defined without reference to what the "true" value or accepted standard value may be. For a group of repeated measurements, if the span of the measured values is relatively small (tightly grouped measurements), the measurements would have high precision. If there is a wide span between the values, the precision will be low. Precision can be expressed in a number of ways. One of the most common is the Standard Deviation from the mean value.

- Selectivity: This is the ability to discriminate between a parameter of interest and possible interferences. Selectivity usually has meaning in the sensing of chemical species where there can be interferences from other chemicals.

- Range: This is defined as the interval over which a parameter is measured--the lowest and highest values of the parameter. 
HNF-3042, Revision 0

- Span: This the width of the range; the difference between the upper- and lower-range values.

\subsection{TEST DESCRIPTIONS}

The tests described in this document address the high priority concerns that were established by a consensus of the PHMC team overseeing and supporting the nested, fixed-depth and at-tank analysis system development and test work (Reich 1998). The tests are presented in the order of their priority and importance in the development, test, and characterization of the nested, fixed-depth fluidic sampling system. As experience is obtained with the nested, fixed-depth sampling system, the testing needs will change. In some cases, additional testing may be required, while in other areas data from previous AEA systems may be sufficient to address the test issues.

\subsection{PHYSICAL BOUNDARY TESTS}

The physical boundary testing will demonstrate that the nested, fixed-depth fluidic sampling system can extract waste samples that are representative of the tank's waste at the sampling inlet, with the sample being

- independent of the elevation of the sampling point in the tank and

- independent of the depth of waste above this sampling point.

This testing will demonstrate that the sampling performance is independent of the physical and chemical properties of the A, B, and C waste envelopes as well as being independent of the waste head and length of a sampling channel. Prior to initiating testing, it will be necessary to ensure that the sampling system hardware is free of residual materials that could influence the test performance. In all cases, it is recommended that the sampling system be purged or thoroughly flushed with water prior to the initiation of a test. If any cleaning agents are used, such as soap, it will be necessary to finish the cleanout with a thorough flush with water. The impact of any residual material should not add more than 0.5 percent error to the test results.

\subsubsection{Sample Point Elevation Test}

The nested, fixed-depth fluidic sampling system will have sampling points (locations of the sampling channel inlets) that range over the full $33 \mathrm{ft}$ of potential waste depth that the 1,000,000-gallon staging tanks can hold. The conceptual design has sample points at approximately the 1-, 3-, 5-, 11-, 15-, 20-, 26-, and 33-ft waste depth positions (Reich 1998). The concern is that the length of a sampling channel may have an impact on the content of an extracted sample. For example, waste taken by the lowest sampling channel (1-ft elevation) may show a different solids content than waste from the highest sampling channel (33-ft elevation), 
HNF-3042, Revision 0

even though the waste depth and content is the same at both of the channel inlets. The impact of waste channel length will be evaluated by comparing the performance of the highest sampling point (33-ft elevation) with that of the lowest sampling point (1-ft elevation) with the same waste head or depth above the sample inlets.

\section{Test Setup:}

The sampling system will be set up to simulate sample extraction from the longest and the shortest sampling channels. For the prototype system, this will be a comparison of the $1-\mathrm{ft}$ and the 33-ft sampling channels.

A single test setup with a two-sample channels or a single-sample channel in two test setups can be used. The test setup should include the valve manifold, valves, sampling station and other system hardware that may impact the acquisition of a waste sample. The sampling channel(s) will be inserted into a simulant vessel with a mechanical mixer that will keep the simulant homogenous during testing (sample acquisition). The vessel will be large enough so that grab samples will not have an effect on the operation and performance of the system. Grab (reference) samples will be taken at the sample channel inlet at the same time a sample is extracted at the sampling point.

Test Simulants:

The simulant for this test will be a slurry mixture of sand and water, with the sand particle size distribution being constant and the water content varying. The sand particles will have a size distribution that is adjusted to be consistent with the high end of that allowed by the expected particle size ranges shown in Table 1. Roughly $1 \mathrm{wt} \%$ of the particles will be in the 500 to $4000 \mu \mathrm{m}$ range, $5 \mathrm{wt} \%$ will be in the 50 to $500 \mu \mathrm{m}$ range, and $94 \mathrm{wt} \%$ will be in the less-than- $50 \mu \mathrm{m}$ range (Rinker 1998a). Two total solids contents will be tested, one with solids in the 1-2 wt\% range and one in the $10-15 \mathrm{wt} \%$ range (the minimum solids specification for the sampler design is $10 \mathrm{wt} \%$, and this should allow some conservatism for mixing errors).

Table 1. Particle Size Range in Phase 1A Privatization Contracts

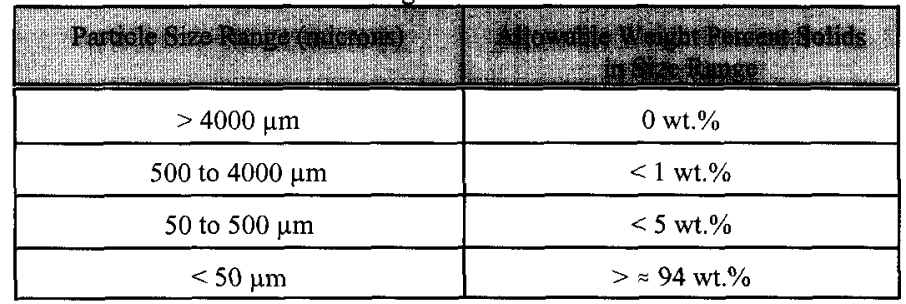


HNF-3042, Revision 0

If greater conservatism is needed, the fraction of large particles shown in Table 1 should be increased rather than selecting an alternative material with higher particle densities. Depending upon the performance with the simulants, it may be recommended that the size of the particles used in the test be either decreased or increased to ensure that the bounding conditions are being examined with the system. It should be noted that the largest expected challenge for the sampling system is the $1-2 \mathrm{wt} \%$ range where the average particle settling velocities will be higher than for the $10-15 \mathrm{wt} \%$ slurry simulant. Since the water will have a lower density and viscosity than the liquid in the tank wastes, the water/sand simulant provides a worst case or conservative test of the performance of the sampling system. A more detailed description and justification of these simulants is included in Rinker 1998a. A summary of tank waste chemical and physical properties can be found in Rinker 1998b.

Test Operation and Performance Analysis:

The volume of the test simulant (and simulant vessel size) will be large enough so that the grab sample has no impact on the content of the simulant (wt\% solids and/or particle content). After a steady-state condition is reached with the simulant and waste being "pumped" through the sampling station, test samples are ready to be taken. The steady-state condition will be when there are no changes in the simulant exiting the sampling system. At the same time a sample is taken with the sampling system, a grab sample will be obtained directly adjacent to the sample channel inlet. The grab sample should be obtained as close to the inlet of the sampling channel as possible in order to ensure that it is sampling the same simulant materials as the inlet of the sampling system is immersed in. Solids and/or particulate analysis may be needed to identify when steady-state has been reached. This procedure will be repeated for both the lowest and highest sampling point setups.

The performance of the sampling system will be assessed by comparing the four samples with each other--two grab samples and the samples from the longest and the shortest sampling channels. The grab samples will provide reference material data that should indicate how well a sampling channel samples the material at its inlet. The measurements made on the samples should include, as a minimum, the particle size distribution and the total $\mathrm{wt} \%$ solids content. Since this test addresses a materials quality issue, five to seven test repetitions are recommended.

\subsubsection{Waste Depth Test}

The depth of waste (head or pressure) above a sample channel inlet may have an impact on the performance of the sampling channel that alters the content of an extracted waste sample. For example, a sampling channel with only $1 \mathrm{ft}$ of waste above its inlet may show a different solids content than a sample extracted when there is $30 \mathrm{ft}$ of waste above the inlet. 
HNF-3042, Revision 0

Test Setup:

The prototype system should be set up to simulate sample extraction from the lowest sampling point ( $1-\mathrm{ft}$ elevation) in the feed tank and the highest sampling point

(33-ft elevation) in a waste tank, which is the largest waste head possible in the feed tank. The waste container that the sampling channel is placed in will be capable of simulating the lowest and highest waste depth expected in the feed tank ( $1 \mathrm{ft}$ to $33 \mathrm{ft}$ ). The simulant vessel will also contain a mechanical mixer that will keep the simulant homogenous during testing (during sample acquisition). The setup must also allow a simultaneous grab sample to be taken near the inlet of the sampling channel for both the highest and lowest waste depths. The simulant vessel should be large enough so that the grab sample does not impact (less than $0.5 \%$ ) the performance of the sampling channels or impact the homogeneity of the sample at the inlets.

Test Simulants:

This test will use the same simulants as the "Sample Point Elevation Test" above. The simulant is a slurry mixture of sand and water with the particle size distribution adjusted to be consistent with the high end of that allowed by the expected particle size ranges shown in Table 1 . The $1 \mathrm{wt} \%$ to $2 \mathrm{wt} \%$ solids range, which is expected to have the largest average settling velocities, should be tested first followed by $10 \mathrm{wt} \%$ to $15 \mathrm{wt} \%$ solids slurry simulant. If greater conservatism is needed, the fraction of large particles shown in Table 1 should be increased rather than selecting an alternative material with higher particle densities. The volume of the test simulant should be large enough so that the grab sample has no impact on the sample wt $\%$ and particle size of the simulant. In addition, the grab sample should be obtained as close to the inlet of the sampling channel as possible in order to assure that it is seeing the same simulant characteristics.

Test Performance Analysis:

In each test setup, it is important that a steady-state condition be reached before test samples are taken. The steady-state condition will have been reached when there are no changes in the simulant exiting the sampling system. At the same time that a sample is taken with the sampling system, a grab sample will obtained directly adjacent to the sample channel inlet. The grab sample should be obtained as close to the inlet of the sampling channel as possible in order to assure it is sampling the same simulant materials as the inlet of the sampling system is immersed in. Solids and/or particulate analysis may be needed to identify when steady-state has been reached. This procedure will be repeated for both the lowest and highest sampling point setups.

The performance of the sampling system will be assessed by comparing the four samples with each other--two grab samples and the samples from the longest and the shortest sampling channels. The grab samples will provide reference material data that should indicate how well a sampling channel samples the material at its inlet. The measurements made on the samples should include, as a minimum, the particle size distribution and the 
HNF-3042, Revision 0

total wt\% solids content. Since this test addresses a materials quality issue, five to seven test repetitions are recommended.

\subsection{CROSS CONTAMINATION TEST}

Cross-contamination of materials is a major concern with the sampling system. In the nested design, each sample channel has its own charge vessel, RFD, and piping that connects to the common valve manifold of the sample bottle station. During operation, this hardware will be full of the material that surrounds the channel's inlet. During operation of the nested, fixed-depth fluidic sampling system, the bottle sample station will be connected to different sampling channels with the valve manifold. During sampling, the sampling station piping, valves, and manifold hardware will all be full of the waste material from this sampling position. When the sampling station is then connected to another sampling channel, there will be residual material within the valve manifold, bottle sample station, and piping that could contaminate the next sample.

Cross contamination is thought to be principally a function of sampler design and operation, but particle settling within the common pipeline and/or adhesion of slurry to the internal surfaces of the sampling system may contribute to cross contamination. The degree of cross contamination is also expected to be related to the ability to flush the sampling channels with waste materials or with externally induced flushing material, such as water. The velocity and density of the flushing fluid is expected to have an impact on the material purging within the sampling system hardware. Potentially, it may be necessary to use a flushing fluid (water) to reduce cross contamination to an acceptable level.

These tests will evaluate the potential for material cross contamination from residual materials within the common valve manifold and bottle sampling station and hardware that extracts samples for all sampling channels and will evaluate the potential for material cross contamination within a sampling channel when the surrounding material has changed. This testing will also demonstrate and help establish the necessary purging that will eliminate or reduce the cross contamination to an acceptable level. Samples will be obtained that provide the decay time for cross contamination when switching from one simulant to another. The source of cross contamination could be within each sampling channel or within the common sampling station hardware connected to the sample channels.

\subsubsection{Sampling Station Cross Contamination}

This test will examine the potential for cross contamination within the sampling station, common to all sampling channels, which contains a valve manifold, sample bottle station, and, potentially, other hardware associated with on-line analysis functions. 
Test Setup:

The hardware for this test will consist of the sample bottle station, two sampling channels, and two simulant vessels. The two simulant vessels will allow each sample channel to be immersed in a different simulant material. The valve manifold will be used to connect the sampling station individually to each sampling channel, simulating in-tank operation. The sampling station will be used to obtain material samples from each of the sampling channels. The simulant vessels will each contain a mechanical mixer to keep the simulant homogeneously mixed during the testing.

Test Simulants:

The simulants that will be used in this cross contamination testing are shown in Table 2-a and Table 2-b. AEA will use a $30 \mathrm{wt} \%$ Kaolin clay-based simulant, indicated in Table $2-a$, to evaluate cross contamination with the conceptual sampling system. The first simulant will be the Kaolin clay/water slurry with the second simulant being pure water. Water is a conservative liquid/supernatant simulant in that its density and viscosity are anticipated to be much smaller in comparison to the actual waste liquid or supernatant constituent.

The cross contamination testing with the prototype system at Hanford will use the simulants shown in Table 2-b. A detailed description and justification for the selection of these simulants is included in Powell and Rinker 1998a and 1998b. The basic difference from the Table 2-a simulants is the amount of Kaolin clay in the slurry. These initial tests at AEA will provide baseline data for continuing the cross contamination testing with the prototype hardware at Hanford.

The first simulant that will be used with the prototype system, shown in Table 2-b, is a thin water/Kaolin slurry. The makeup of this simulant will be a $25 \mathrm{wt} \%$ EPK Pulverized Kaolin clay from Feldspar Corporation (Edgar, Florida) mixture with water. The $25 \mathrm{wt} \%$ solids slurry will have a density of about $1.18 \mathrm{~g} / \mathrm{mL}$ and a yield stress of about $2.0 \mathrm{~Pa}$. Although this is a relatively small yield stress, the tank waste materials are likely to have even smaller yield stresses. Therefore, this simulant is expected to represent a worst-case scenario where there is sample adherence to the surfaces of the sampling system hardware.

The solids concentration and yield stress of an actual tank waste sludge layer are expected to be much greater than those offered by the $25 \mathrm{wt} \%$ water $/ \mathrm{Kaolin}$ slurry simulant. To evaluate the potential for cross contamination with a sludge material, a $45 \mathrm{wt} \%$ water/Kaolin slurry will also be used, as indicated in Table 2-b. This simulant will provide cross contamination data for evaluating the condition when the sampling channel is immersed in a waste sludge layer that will be diluted later to a relatively thin liquid or supernatant material. The $45 \mathrm{wt} \%$ water/Kaolin slurry will have a yield stress of 
HNF-3042, Revision 0

approximately $60 \mathrm{~Pa}$ (in the range of a concrete slurry), which is similar to that measured in tank sludge samples that have been recently disturbed (such as the sluicing/pumping actions).

Table 2-a. Simulant Compositions for Cross-Contamination Testing with the Conceptual Sampling System.

\begin{tabular}{|l|l|}
\hline 1) Kaolin Clay/Water Slurry & $30 \mathrm{wt} \%$ Kaolin Clay in Water \\
\hline 2) Water & Clean Water \\
\hline
\end{tabular}

Table 2-b. Simulant Compositions for Cross-Contamination Testing with the Prototype Sampling System.

\begin{tabular}{|l|l|}
\hline 1) Water/Kaolin Slurry & $\begin{array}{l}25.0 \mathrm{wt} \% \text { EPK Pulverized } \\
\text { Kaolin in Water }\end{array}$ \\
\hline 2) Water/Kaolin Thick Slurry & $\begin{array}{l}45 \mathrm{wt} \% \text { EPK Pulverized Kaolin } \\
\text { in Water }\end{array}$ \\
\hline 3) Water & Clean Water \\
\hline
\end{tabular}

Sample Acquisition and Analysis:

Initially, the sampling station will be connected to the first sampling channel which is immersed in the clay/water slurry simulant. The system will be operated until a material steady-state condition is reached, and there is a consistent material flow through the sampling station. During this time, the discharge flow from the sampler can be directed back into the simulant reservoir. A sample will be extracted with the sampling station and used as a reference to provide particulate and solids content performance for the first simulant in case the sample channel and sampling station modify the particulate distribution and solids content. The sampling station will then be connected to the second sample channel that is immersed in water. The discharge from this flow shall not be directed back into the simulant reservoir because it will bias the simulant's characteristics. Prior to initiating this test, the number of pump cycles to complete materials changeover (less than $1 \%$ residual of the previous simulant) will be estimated (or a purge pre-test completed and pump cycles tallied). Samples will be taken at pre-selected time intervals as the second simulant (water) replaces the first simulant (water/clay) in the sampling system. At a minimum, samples will be obtained after reaching approximately $1 / 4,1 / 2,3 / 4,1$, and $2 x$ of the total number of pumping cycles required to reach a steady-state condition (less than $1 \%$ residual of the previous simulant present). 
The impact of cross contamination will be assessed by comparing the contents of the extracted samples with the water simulant. A comparison of these measurements with the reference sample data will provide an indication of the potential for cross contamination. Since the particulate size distribution for the Kaolin and clay simulants is probably too small for inertia to have much impact, measuring total solids content will be sufficient. The analysis of the residual materials in the samples should indicate the potential need to (or the conditions under which it may be necessary to) actively flush the sampling system with a flushing fluid (water) injected into the sampling system.

The initial analysis will be made by comparing the opacity of the samples with the second (water) simulant. A more detailed analysis can be made by plotting the particle size data and total waste volume as a function of the number of pump cycles accumulated when the sample was extracted. This will establish a "decay" curve for the elimination/reduction of cross contamination. The number of pump cycles required to reduce cross contamination to an acceptable level is an important parameter in the operation of the sampling system. Since these test simulants represent worst case waste properties/conditions, the cross contamination decay envelope is expected to be a bounding limit for operation with real tank wastes. Since this test addresses a materials quality issue, five to seven test repetitions are recommended.

\subsubsection{Sample Channel Cross Contamination}

This test will examine the potential for cross contamination due to residual waste material within a sampling channel. Each sampling channel contains its own charge vessel and RFD, in addition to interconnecting piping. This test will be completed only with the prototype sampling system at Hanford.

Test Setup:

The test setup will consist of the sampling station connected to a single sampling channel and two sample vessels that will be filled with different simulants. Although reference samples will be obtained, it will be important to minimize potential errors due to simulant settling or layering that may result in a change of particulate distribution or total solids content. The simulant vessels will have a mechanical based mixer that keeps the simulants homogeneous, particularly around the sample channel inlet. The vessel's volume must be large enough to contain enough water to complete the cross contamination test. When the sampling channel is in the second vessel, the outlet from the sampling station will not be cycled back into the simulant vessel. 
Test Simulants:

The simulants that will be used in this cross contamination testing are the same simulants used in the Sampling Station Cross Contamination tests, shown in Table 2-b. Water will also be used as the comparison. The $30 \mathrm{wt} \%$ clay (Table $2-\mathrm{a}$ ) and the $25 \mathrm{wt} \%$ Kaolin (Table 2-b) simulants will provide cross contamination test data where there is a materials adherence to the surfaces of the sampling system hardware. The $45 \mathrm{wt} \%$ Kaolin simulant provides similar cross contamination data for evaluating the condition when the sampling channel is immersed in a waste sludge layer that will be diluted later to a relatively thin liquid or supernatant material. Water is a conservative liquid/supernatant simulant in that its density and viscosity are anticipated to be much smaller in comparison to the actual waste liquid or supernatant constituent.

Sample Acquisition and Analysis:

After completing system flushing/purging, the first simulant vessel will be filled with the first simulant (the $30 \mathrm{wt} \%$ clay or the $25 \mathrm{wt} \%$ Kaolin) and the vessel mixer activated to maintain a homogeneous material around the sampling channel inlet. The sampling system will be run until a steady-state condition is reached (the material being pumped through the sampling system is homogeneous). The material flow to reach this steady state can be returned to the simulant vessel. A sample will then be extracted with the sampling station. This will serve as a materials reference (particulate distribution and $w t \%$ solids) if the sampling system alters the simulant content.

The sampling channel will then be removed from the first simulant vessel and placed in the second simulant vessel that contains only water. It will be necessary to wash down the outside of the sampling channel before beginning the test. However, the inlet should be plugged before the sampling channel is removed from the first simulant vessel. The second simulant vessel must contain enough water to complete testing, as the outlet from the sampling system will not be returned to the vessel. The system operation will then be resumed and sampling at the pre-selected times immediately initiated as the water begins to purge/replace the residual simulant in the sampling system. As indicated above, the number of pump cycles to complete materials changeover (less than $1 \%$ residual of the previous simulant) will be estimated (or a purge pre-test completed and pump cycles tallied). At a minimum, samples will be obtained after reaching approximately $1 / 4,1 / 2$, $3 / 4,1$, and $2 \mathrm{x}$ of the total number of pumping cycles required to reach a steady-state condition (less than $1 \%$ residual of the previous simulant). A comparison of opacity can be used as an initial indicator of cross contamination. However, total solids content (and particulate distribution) measurement is much more accurate. Although the second simulant will be water, a grab, reference sample, will be taken near the sampling system at the same time that the last sample is extracted by the sampling system.

The decay of the cross contamination will be obtained by plotting the total solids content (opacity) as a function of the number of pump cycles accumulated when the sample was extracted. The number of pump cycles required to reduce cross contamination to an 
HNF-3042, Revision 0

acceptable level is an important parameter in the operation of the sampling system. Since these test simulants represent worst case waste properties/conditions, this cross contamination decay envelope is also expected to be a bounding limit for operation with real tank wastes. The analysis should indicate the potential need to, or the conditions under which it may be necessary to, actively flush the sampling system with a flushing fluid

(water) injected into the sampling system. Since this test addresses a materials quality issue, five to seven test repetitions are recommended.

\subsection{WASTE PHYSICAL PROPERTIES}

This test will provide data that demonstrates that the waste samples extracted by the nested, fixed-depth fluidic sampling system are representative of the waste at the inlet of the sampling channel.

The tank wastes will contain both liquids and solids that are in the form of insoluble as well as soluble solids. If no solids are present and the liquid is not in a near saturated state, the extracted liquid sample should not differ from the solid-free liquid surrounding the sampling channel inlet. When a steady-state condition is reached with a sampling channel, the sampling hardware should maintain the same temperature as the waste moving through it. Therefore, there should be no problems with the precipitation of materials in the sampling system. However, when solids in the form of different sized particles are present, there are several mechanisms that might result in a non-representative sample. These include the following:

- Particle settling due to density changes of the solid and liquid phases of the waste,

- Selective particle, capture, transportation and sampling due to particle size, shape, and density differences, and

- Changes in the viscosity of the liquid constituent carrying the particles.

A more detailed description of the potential mechanisms for producing non-representative sampling with the nested, fixed-depth fluidic sampling system is included in Powell and Rinker 1998a.

Test Setup:

The setup for this test will consist of a single sampling channel connected to the sampling station with all of the hardware representative of an in-tank sampling system (the sampling station including all components such as valves, valve manifold, and sample bottle fixture). To conserve resources and time, it is recommended that this test be run in conjunction with the Physical Boundary Tests described in Section 5.1 above.

The dimensions of this hardware will be representative of the system that is being developed for in-tank deployment. The sampling channel will be set up to emulate the 
HNF-3042, Revision 0

lowest sampling point (1-ft elevation) anticipated in a waste tank (largest separation between the sample channel inlet and the sampling station). The longest sampling channel should provide the greatest potential for particulate settling between the inlet and the bottle sampling point and other problems with sampling performance. The simulant vessel, in which the sample channel is mounted, will contain a mechanical mixer to keep the simulant in a homogenous state. The simulant vessel will also be large enough to complete grab sampling (reference material) adjacent to the sample channel inlet without impacting the homogeneity of the simulant or the performance of the sampling channel.

Test Simulants:

The simulants for this test will be a slurry mixture of sand and water with the sand particle size distribution as shown in Table 1 above. These are the same simulants described in Section 5.1 above for the Physical Boundary tests. The simulants will have a sand content with a size distribution adjusted to be consistent with the high end of that allowed by the expected particle size ranges shown in Table 1 . Roughly $1 \mathrm{wt} \%$ of the particles will be in the $500 \mu \mathrm{m}$ to $4000 \mu \mathrm{m}$ range, $5 \mathrm{wt} \%$ will be in the $50 \mu \mathrm{m}$ to $500 \mu \mathrm{m}$ range, and $94 \mathrm{wt} \%$ will be in the less-than- $50 \mu \mathrm{m}$ range. Two total solids contents should be tested, one with solids in the $1 \mathrm{wt} \%$ to $2 \mathrm{wt} \%$ range and one in the $10 \mathrm{wt} \%$ to $15 \mathrm{wt} \%$ range (the minimum envelope solids content for the sampler design is $10 \mathrm{wt} \%$, and this should allow some conservatism for mixing errors). Since the water will have a lower density and viscosity than the liquid in the tank wastes, the water/sand simulant provides a worst-case or conservative test of the performance of the sampling system.

If greater conservatism is needed, the fraction of large particles shown in Table 1 should be increased rather than selecting an alternative material with higher particle densities. Depending on the sampling performance, it may be recommended that the maximum particle size tested either be decreased or increased to ensure that the bounding conditions are being examined with the system. It should be noted that the largest expected challenge for the sampling system is the $1 \mathrm{wt} \%$ to $2 \mathrm{wt} \%$ range where the average particle settling velocities will be higher than for the $10 \mathrm{wt} \%$ to $15 \mathrm{wt} \%$ slurry simulant. A more detailed description and justification of these simulants are included Powell and Rinker 1998a and 1998b.

The volume of the test simulant should be large enough so that the grab sample has no impact on the sample wt $\%$ and particle size distribution of the simulant. In addition, the grab sample should be obtained as close to the inlet of the sampling channel as possible in order to ensure that it is sampling the same simulant materials.

Sample Acquisition and Analysis:

Sample acquisition and analysis will be the same as described for the Physical Boundary tests in Section 5.0 above. After setup, the sampling system will be operated until a material steady-state condition is reached, and there is a consistent, homogeneous flow of 
simulant through the sampling station. A reference grab sample will be taken at the same time that a sample is extracted with the sampling station. Since this test concerns material quality, a five to seven test repetition with each simulant material is recommended. This procedure will be repeated with each simulant recipe.

All samples, including the grab samples, will be analyzed for particulate size distributions and total solids content. A comparison of the particle size distribution and the total solids content data from the bottle samples with similar data from the grab samples will indicate the performance of the sampling system.

\subsection{STARTUP AND OPERATION FROM A PLUGGED CONDITION}

This test will demonstrate that the sampling system can recover from credible stoppages or plugged conditions caused by the tank wastes. Recovery will be deemed successful if, after the stoppage or plugging is mitigated, representative samples can be obtained with the sampling system.

The physical and chemical property specifications for waste envelopes A, B, and C are limited to a relatively narrow range which are characteristic of supernatant wastes with a fairly dilute solids content (less than $10 \mathrm{wt} \%$ ). The tank wastes, from which these supernatant wastes will be extracted, will have a wider range of chemical and physical specifications. To bring some of the waste feeds into compliance with the A, B, and C envelopes, dilution and settling may be required. Since the sampling system inlets will span the full depth of waste in a tank, they can be located in wastes with significantly different solids content than in these envelopes. More details on the range of chemical and physical properties of anticipated wastes can be found in Powell and Rinker 1998b.

Although accurate waste sampling may not specifically be needed for wastes that are outside the A, B, and C envelopes, the sampling system must be capable of reliably operating (pumping the materials through the sampling station) with all of the material anticipated to be in the feed tank, including a relatively high solids content waste. During operation with these materials, the sampling hardware could become plugged either from the settling of suspended particles or from the precipitation of saturated solutions. The potential for plugging will increase if the system operation is halted while it contains some of these wastes, or if the temperature of the waste drops.

The sampling system must be capable of recovering from a stoppage or plugged condition and subsequently extracting a representative waste sample. This test addresses both precipitation- and settling-based stoppage or plugging conditions. It also provides opportunity for the testing of preventive methods, such as back flushing and purge flushing before a system is shut down. If procedures are developed for implementing purging or flushing for 
HNF-3042, Revision 0

recovery/mitigation of stoppage/plugging, this test will be used to validate the recovery of sampling performance.

\section{Test Setup:}

The setup for this test will consist of a single sampling channel connected to the sampling station with all of the hardware representative of an in-tank sampling system--the sampling station including all components such as valves, valve manifold, and sample bottle fixture. The system should contain all full-size hardware and all of the flushing options anticipated with the in-tank sampling system. The system should be set up to emulate a sample channel elevation which is anticipated to have the greatest potential for plugging or stoppages. The lowest sampling inlet ( $1 \mathrm{ft}$ elevation) will have the highest potential for plugging or stoppage, as it will be at the bottom of a settled waste volume (have the highest percentage of large particles and have the highest solids content).

The vessel containing the test simulant and sampling channel will contain a mechanical mixer that will be used to keep the simulant in a homogenous state. The simulant vessel will also be large enough to complete grab sampling adjacent to the sample channel inlet without impacting the homogeneity of the simulant or the sampling performance. A grab sample will be taken at the same time the sampling system is taking a sample with the bottle in the sampling station. The grab samples will provide reference material for comparison with the materials in the bottle samples.

Test Simulants:

As shown in Table 3, two simulants, one for precipitation-based plugging and one for settling-based plugging, will be used for testing startup and operation from a plugged condition. A detailed description and justification for the simulants can be found in Powell and Rinker 1998a.

Table 3. Simulants for Evaluating Recovery From Plugged Conditions

\begin{tabular}{|l|l|}
\hline \multicolumn{1}{|l|}{} & \\
\hline \hline Precipitation Plugging Simulant & $\begin{array}{l}\text { Coarse-grained K-Mag with } \\
\text { approx. 1 wt\% water cured 24 hours }\end{array}$ \\
\hline Solids Settling Plugging Simulant & $\begin{array}{l}30 \text { wt\% Min-U-Sil 30 Silica Flour } \\
\text { in water }\end{array}$ \\
\hline
\end{tabular}

Precipitation-plugging Simulant:

The simulant to test plugging caused by crystal formation from precipitation, shown in Table 3, is a mixture of coarse-grained K-Mag fertilizer with $1 \mathrm{wt} \%$ water. Based on tank waste data, the shear strength for a precipitant-plugging simulant should be in the $25 \mathrm{kPa}$ range (Powell and Rinker 1998a). The water and K-Mag granules should be mixed until 
HNF-3042, Revision 0

the water is uniformly distributed. Then the moistened K-Mag should be packed into the place where plugging is anticipated and allowed to cure for 24 hours. Some sort of seal needs to be made on both sides of the K-Mag to keep the water from evaporating. The compressive strength of the $1 \%$ water $/ 99 \% \mathrm{~K}-\mathrm{Mag}$ will be in the $7 \mathrm{psi}$ range after 24 hours of curing. This implies a shear strength on the order of $25 \mathrm{kPa}$. (For comparison purposes, the shear strength of frozen margarine is approximately $50 \pm 20 \mathrm{kPa}$ [Powell et al. 1997]).

This material is soluble in water, but only slow soluble relative to rapidly soluble salts such as sodium nitrate that will be found in the tank waste. The mechanical strength of the K-Mag results from a hydration reaction that binds the particles together. The strength of the cured simulant can be controlled by varying the water content in the initial mixture. The strength of this plugging simulant is likely greater than the strength of an actual waste plug, but is an upper bound. The strength of the waste plugs is difficult to establish because the needed waste physical property data are not available.

Solids Settling Plugging Simulant:

The recommended simulant for testing plugging as a result of particulate settling is a mixture of $30 \mathrm{wt} \%$ Min-U-Sil 30 Silica Flour with water, as indicated in Table 3. When completely settled, this simulant should form a settled bed of particles with a high mechanical strength and low permeability. The simulant particles should be small, but larger than 5 microns (Powell and Rinker 1998a). The solids concentration in the initial slurry is not critical, but it should represent the largest solids concentration that will be experienced with the tank wastes. For this test simulant, the solids content will be about $30 \mathrm{wt} \%$. This represents a compromise between the high solids content anticipated to be found in the real wastes and the particulate settling which may be hindered at higher concentrations of solids.

A plugged condition is set up by filling the sampler with this simulant and allowing it to settle. Initially a settling time of 28 hours is recommended, but 24 hours is probably adequate. Prior to testing the recovery of the system, the settling properties of this simulant should be pretested and verified. For more accurate guidance, a tall clear cylinder should be filled with the simulant and the thickness of the settled bed charted as a function of time (location of the solids/water interface).

Test System Preparation, Operation, and Data Acquisition/Analysis:

Two test campaigns will be required--one to evaluate recovery from precipitation-based plugging and one to evaluate recovery from settling-based plugging. Initially, samples of both simulant mixtures should be prepared and settled or precipitated and the resulting solid analyzed to verify that it has the appropriate solid or plugging properties. The amount of solid formed relative to the initial slurry volume should be measured. The relative strength of the solids may also be measured to assure that the solid is within the range of mechanical properties anticipated with real wastes. 
HNF-3042, Revision 0

AEA will identify areas where plugging from settling and precipitation is most likely expected to occur. An initial plug on the order of 0.5 to 1.0 inch deep/long should be set up initially at these locations. Then the purging/cleaning process should be started and continued until the plug is broken. The number of cycles, pressure, flow, etc., required to break through the plug, will be recorded. After breakthrough is detected, the system will be cycled to complete the purging/cleaning process using water, and the number of sampling system cycles required to recover and operate normally will be recorded.

The size of the plug will then be increased by $0.5-1.0$ inches and this process repeated until the purging/cleaning process in no longer able to break through the plug. This process will be repeated for each point in the sampling channel that is identified as a possible plugging site and the appropriate settling and/or precipitation simulant used.

If the plugging can be accurately characterized, and care is exercised in obtaining and analyzing samples, a single test with each size plug may be sufficient. If error is suspected as a result of non-conforming test data, then the test should be repeated.

\subsection{LIFE CYCLE EVALUATION}

This testing and/or evaluation will demonstrate that the nested, fixed-depth sampling system can successfully operate over an expected 20-year lifetime. During this lifetime, the sampling system must be capable of extracting waste samples that are representative of the wastes that the system is deployed in.

Tank wastes are made up of highly caustic salt solutions that contain soluble and insoluble solids in the form of particles and crystals. Some of the lower sampling channels will experience waste slurries with physical and chemical properties that are outside of the privatization contract envelope. With some of the tank wastes, static conditions can result in the settling of suspended particles, while changes in $\mathrm{pH}$ and temperature can produce precipitation of solids. These can be in the form of very abrasive crystals and particles. Over the expected lifetime of the sampling system, some of the system's components, such as the reverse flow diverters (RFDs), valves, valve manifolds, elbows, tees, etc., may experience wear/erosion that adversely impacts the performance and operation of the sampling system. Erosion is expected to take place where there are high waste velocities and high shear forces. In addition, precipitated solids and settled solids that are entrained between moving surfaces (valves, etc.) can produce erosion and wear that could also affect the sampling system's operation.

The objective of this testing is to produce accelerated abrasion in the sampling system. However, archived data from previous sampling system applications and testing may be available to verify the expected lifetime of the sampling system. It is preferred that archived data be made available either in place of accelerated testing or to augment testing. 
Test Setup:

The lifetime testing could be very destructive to a sampling channel and should be completed with a very simple, expendable experimental setup that uses a single sampling channel. The test setup would consist of a single sampling channel connected to a mockup sampling station. The sampling station would consist of two valves connected so that the simulant could be ported to two flow paths. One would have a bottle sampling station, and the other would be just a pipe segment. This will allow the valves and manifold to be exercised with the abrasive simulant. The system should contain all full-size hardware and a means to flush the system for completing visual examination of points where accelerated wear is anticipated. The test system should be set up for continuous operation or cycling.

Simulant for Lifetime Testing:

The simulant recommended for abrasion testing is a water/silicon carbide slurry. If possible, the particle size should cover the range shown in Table 1 above, as there might be preferential paths in the sampling for the different sized particles. It is recommended that a $15 \mathrm{wt} \%$ silicon carbide slurry be used. During testing, it may be necessary to replenish the simulant water content if evaporation is an issue. After each test interval is completed, the simulant should be replaced with fresh water and new silicon carbide abrasive.

Test System Preparation, Operation, and Data Acquisition/Analysis:

The simulant vessel will be filled with simulant and a mixer pump used to maintain the homogeneity of the simulant. After steady-state conditions are reached and a suitable silicon carbide content achieved, the sampling system will be operated for several extended time periods. During the total test time period, it is critical that the operation of the system not change, such as the air pressure, cycle time, etc. Based on an expected 15 -year lifetime, a total accumulated operating time (estimated number of pumping cycles) for the sampling system will be determined. The system operation will then be halted at time periods representing $1 / 4,1 / 2,3 / 4$, full, and $1 \frac{1}{2}$ of the total expected operating cycles. During operation, the valves should be activated to represent sampling. For each operating time period, the performance of the system will be determined by recording the following:

- the volume of simulant pumped in one system cycle,

- the liquid velocity at the outlet of the sampling station, and

- the pressure cycle at the bottle station.

In addition, a bottle sample will be extracted for reference purposes at the beginning and end of each test period. A comparison of the performance data will indicate any changes in system operation due to erosion.

After each operating time interval, the system will be shut down and flushed and critical surfaces examined/measured to assess the level of erosion. Photographs will be made of 
HNF-3042, Revision 0

critical components and surfaces where wear/erosion is expected. The data accumulated after each operating period will be examined to identify any evidence of progressive component erosion and degradation of the sampling system operation. This data will be used in considering mitigation measures that will assure consistent sampling performance over the lifetime of the sampling system. Since this test is expected to be cost- and time-limited, a single, carefully orchestrated and documented test is recommended. Archived lifetime data should be used in the planning of this testing.

\subsection{SAMPLING SYSTEM IN-TANK ORIENTATION}

These tests will demonstrate or show that the sampling system can extract representative samples without interference with in-tank mixing operations.

A large, 300-hp mixer pump will be installed in the feed tank to agitate and mix the waste. The direction of the flow from this pump will be changed to continuously re-direct the flow within the tank. There will be incidences when this pump flow will impinge directly upon the sampling hardware and potentially upset the performance of a sampling channel. The back pressure on an RFD may be changed, or the flow around the sampling hardware could create shear forces that impact the material homogeneity in the vicinity of an inlet nozzle (change in particulate size distribution). The objective of this testing is to determine the potential impact of flow on the operation and performance of the sampling system hardware and then to identify and test any measures required to mitigate undesired performance changes. This test does not address any structural impacts or concerns for the sampling hardware that will experience the effluent from the mixer pump.

Test Setup:

The hardware for this test will consist of a single sampling channel, sampling station, and simulant vessel with a pump that can produce material flow levels expected in the feed tank. The simulant vessel should also have a mixer pump to maintain the homogeneity of the simulant during testing. The pump outlet and sampling channel will be set up to simulate the flow conditions expected in the feed tank. The tests should include the following flow/pump conditions:

- no flow from the pump (this is the comparison or reference condition),

- $\quad$ pump flow directly into a sampler inlet (0 degree position),

- $\quad$ sample nozzle 90 degrees to pump flow, and

- $\quad$ sample nozzle 180 degrees to pump flow.

Test Simulant:

The test simulant recommended for this test will be the sand/water slurry mixture used in the Physical Boundaries Tests, described in Section 5.1 above. The sand particles should have a distribution adjusted to be consistent with the high end of that allowed by the expected particle size ranges shown in Table 1. The total solids content should be in the 
HNF-3042, Revision 0

$10 \mathrm{wt} \%-15 \mathrm{wt} \%$ range, which provides a worst-case condition for solids. The volume of the test simulant should be large enough so that the sampling has an insignificant impact on the test conditions.

Test System Preparation, Operation, and Data Acquisition/Analysis:

The simulant vessel will be filled with the simulant and the mixer pump activated. After steady-state conditions are reached, the system is ready for testing. The sample channel performance will be tested for the four setups described above. In each test setup, the following will be recorded:

- the volume of simulant pumped in one system cycle,

- the liquid velocity at the outlet of the sampling station, and

- the pressure cycle at the bottle station.

A bottle sample will also be taken for each of the four flow conditions. A reference sample will be taken when the mixer pump is off. For analysis, a comparison of the volume, flow, and pressure data with the no-flow data will indicate any changes in system operation due to material flow from the mixer pump. If there are changes, the constituents of the sampled material (particle size distribution and total wt $\%$ of solids) will be analyzed to assess any potential impact on sampling performance. The tests will be repeated with each mitigation measure to assess and demonstrate its effectiveness. Since this test addresses a materials quality issue, five to seven test repetitions are recommended.

\subsection{IN-TANK DEPLOYMENT AND OPERATION TESTING}

Safety requirements limit the size and weight of the hardware deployed in or on a waste tank. The load limitations for hardware deployed in each Hanford tank are usually unique because of design differences and other factors such as tank aging. For each of the feed tanks (AP-102 and AP-104), a 12-inch riser has been identified and "reserved" for exclusive use by the nested, fixed-depth sampling system. Testing and/or analysis is needed to assure that the sampling system can now be safely deployed and safely operated over the anticipated contract time. Engineering calculations and analyses may be sufficient in lieu of testing for some of the test issues described below.

Deployment Testing

Testing or analysis will be completed that demonstrates the following:

- The diameter of the in-tank sampling system hardware is within acceptable dimensions for the selected tank risers. The current limit is 10.5 inches maximum outside diameter for the sampling system.

- The sampling system can be raised from a horizontal position to a vertical position (ready for riser insertion) and installed in the riser using cranes. 
HNF-3042, Revision 0

- Deployment in the riser can be completed without damage to the riser or to other tank hardware.

- The static load from the deployed system that must be supported by the tank is within a tank's acceptable limits for the selected riser and tank dome. This includes the sampling system weight and the weight of any entrained wastes.

Operational Testing:

This testing or analysis will demonstrate that the sampling system will not be impacted by the operation of other in-tank hardware. The testing will provide data that shows the fill rate and fill level with the $500 \mathrm{ml}$ sample bottle. Testing or analysis will be completed that demonstrates the following:

- The dynamic loading from the sampling hardware can be safely supported by the tank or tank riser, such as the reactive loading generated by the cyclic/pulsing movement of waste through the sampling system.

- The tank can withstand any secondary force, shock, or vibration from the sampling system that is a result of other tank operations, such as the side load resulting from the mixer pump effluent impinging on the in-tank hardware, vibration from pumps, etc.

- The sampling system hardware can safely withstand any force, shock, or vibration imposed upon it by the tank

- The exposed tank components will not be adversely impacted by natural weather conditions (wind, dust, moisture, freezing temperature, and temperatures exceeding $100^{\circ} \mathrm{F}$ ).

- The sample bottle filling rate as a function of "pump" cycles and/or sampling time.

- Demonstrate that the sample bottle can be transferred from the sample chamber to the site's shipping cask within acceptable safety and exposure limits.

\subsection{TEST DOCUMENTATION}

Uniquely labeled laboratory notebooks will be used to record all test data. This test data will include test description/objectives, test system setups, a description of serial numbers and/or identification numbers for all sensing/measurement systems and/or tools used in the tests, the measurement and test data, and any in-test calculations made. These notebooks will have numbered pages. Entries will be made in ink, with any entry changes crossed out but not erased. The calibration status of any measurement tool or system will also be recorded.

All data recording media, such as computer disks and strip charts, will be clearly and uniquely identified and stored in a manner to preserve the recorded data. The notebooks will record the identification of the storage media that is used for each test. 
HNF-3042, Revision 0

At the conclusion of the testing, a written test report will be prepared. This report will summarize test purposes and condition, test setups, test data and processed test data, along with conclusions resulting from the completion of the test and reference data extracted from the laboratory notebook.

\subsection{ORGANIZATIONAL RESPONSIBILITIES}

The PHMC Team has the responsibility for defining all tests, identifying the simulants, and developing the test acceptance/rejection criteria for all testing that will be completed with either the conceptual sampling system or the full-scale prototype system. The Engineering Task Plan identified the roles and responsibilities for these testing activities (Reich and Smalley 1998).

AEA has the responsibility for performing the necessary design and proof-of-principle testing for the conceptual nested, fixed-depth sampling system. The data, including the notebooks, will be submitted to the PHMC team along with a written summary of the tests completed. The PHMC will have the responsibility for the testing of the prototype system at the Hanford cold test facility.

\subsection{QUALITY ASSURANCE}

The measuring devices used in these tests will be calibrated or characterized before use in any of the tests described in this test plan. Prior to initiating testing, documentation will be available for each measuring device that describes its measuring functions, the property to be measured, and the test/calibration data that shows the performance (accuracy and precision) of the measuring device. Should calibration be required, secondary standards are deemed accurate enough to quantify the performance of the sampling system in this testing.

\subsection{DOCUMENTATION}

All nested, fixed-depth sampling system testing documentation will be released into the PHMC document control system. Responsibilities, policies, and procedures for so doing will follow those described in the Engineering Task Plan (Reich and Smalley, 1998). 
HNF-3042, Revision 0

\subsection{REFERENCES}

DOE, 1997a, DE-AC06-RL13308, BNFL Inc. Privatization Contract, U.S. Department of Energy, Richland Operations Office, Richland, Washington.

DOE, 1997b, DE-AC06-RL13309, Lockheed Martin Advanced Environmental Systems Privatization Contract, U.S. Department of Energy, Richland Operations Office, Richland, Washington.

HNF-2019, Rev. 0, 1998, Critical Risks in Tank Waste Remediation System Retrieval and Disposal Mission Readiness-To-Proceed Memorandum, Fluor Daniel Hanford, Inc., Richland, Washington.

HNF-SD-WM-TRD-007 Draft, Rev. 0, 1998, System Specification for the Double-Shell Tank System, Fluor Daniel Hanford, Inc., Richland, Washington.

Meyer, S. L., 1975, Data Analysis for Scientists and Engineers; New York: John Wiley and Sons, New York, New York.

Reich, F. R., 1998, Meeting Minutes: Variable Depth Sampler Team Meeting - April 21, 1998, COGEMA Engineering Corporation, Richland, Washington.

Reich, F. R., and J. L. Smalley, 1998, HNF 2056, Rev. 0, Engineering Task Plan for Development, Fabrication, and Deployment of Nested, Fixed-depth Fluidic Sampling and At-Tank Analysis Systems, COGEMA Engineering Corp., Richland, Washington.

Rinker, M. W. and M. R. Powell, 1998a, Simulant Specification for Nested Fixed-Depth Sampler, (external letter No. 9854073), Pacific Northwest National Laboratory, Richland, Washington.

Rinker, M. W. and M. R. Powell, 1998b, Preliminary Set of Chemical Analytes and Physical Properties for Waste Monitoring, (external letter No. 9854071), Pacific Northwest National Laboratory, Richland, Washington. 
HNF-3042, Revision 0

\section{APPENDIX A \\ FLUIDIC BASED SAMPLING SYSTEM COMPONENT DESCRIPTION}


HNF-3042, Revision 0

\section{APPENDIX A - FLUIDIC BASED SAMPLING SYSTEM COMPONENT DESCRIPTION}

\section{A.1 SAMPLER GENERAL DESCRUPTION}

A fluidic sampler consists of two main components, the fluidic Reverse Flow Diverter (RFD) Pump, and the fluidic sampling Tee. Both components are integral to the sampler operation: the pump is used to lift the sample to the Tee, and the Tee draws the sample into the sampling bottle. The main elements of a fluidic RFD pump are shown in Figure A-1 and consist of the following:

- pumping element - a passive fluidic device through which fluid enters the pump from the supply tank. The details of this element are described later.

- charge vessel

- primary controller for providing the gas pressure and flow conditions in the charge vessel and acting as a barrier between the clean incoming compressed gas and the potentially hazardous liquid

- secondary controller which handles only clean gas and provides the gas flow to the primary controller as required

\section{A.2 OPERATIONAL CYCLE}

The pump typically operates in three phases as illustrated below:

Refill Phase:

In this phase, liquid from the supply tank enters the charge vessel through the pumping element, and the phase continues until the liquid level has reached the top of the charge vessel and enters the air link pipe. When required, a partial vacuum is applied to the charge vessel by the primary controller to augment the filling rate.

Drive Phase:

In this phase, compressed gas is passed via the primary controller into the charge vessel which forces the liquid through the pumping element and along the delivery pipe into the delivery vessel. This continues until the air-liquid interface reaches the bottom of the charge vessel, at which time the compressed gas supply is terminated. The role of the pumping element in this phase is to entrain further fluid from the supply tank into the delivery pipe or, at the very least, to minimize the amount of fluid flowing back into the supply tank.

Vent Phase:

In this, the third and final phase, the compressed gas supply to the pump has ceased, and the high pressure gas in the charge vessel is allowed to escape to vent through the primary controller. The liquid contained in the delivery pipe also tends to fall back into the charge vessel. The amount by which the pipe empties depends upon the pump design, the imposed operating frequency, and the type of pump element used. When the pressure in 
the charge vessel has fallen close to atmospheric pressure, the refill phase recommences, and the whole cycle is repeated.

The pump therefore operates in a cyclic manner, delivering intermittent "dollops" of liquid into the delivery vessel.

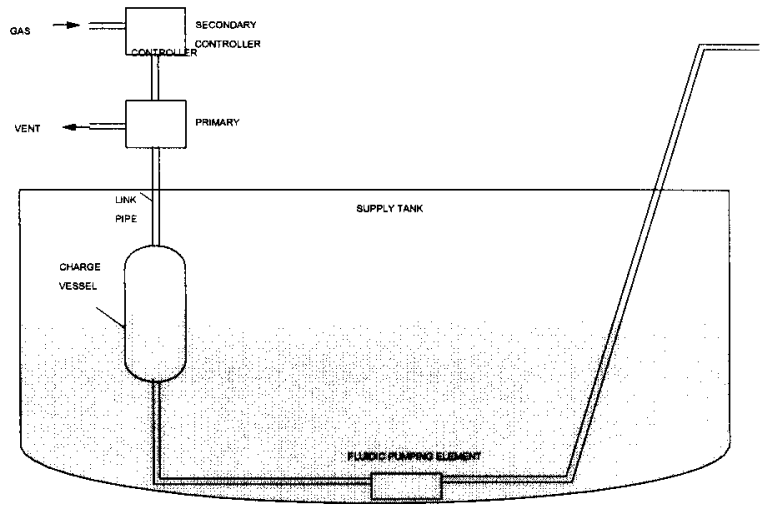

Figure A-1. Schematic Showing the Main Elements of a Fluidic RFD Based Pump. 
HNF-3042, Revision 0
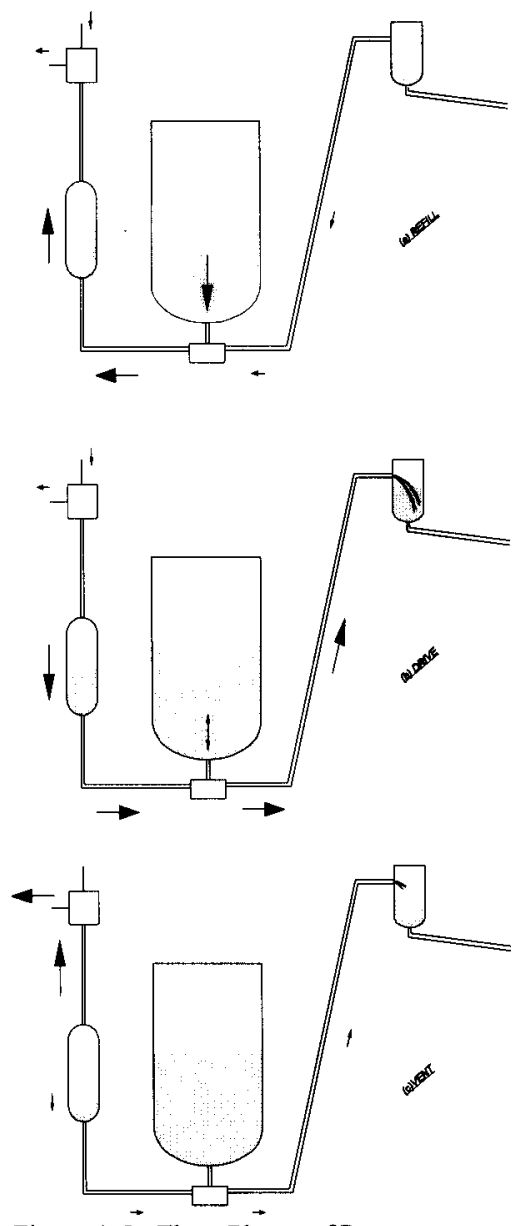

Figure A-2. Three Phases of Pump

Operation; Top to Bottom, Refill, Drive, and Vent 


\section{A.3 PUMPING ELEMENTS}

\section{A.3.1 Reverse Flow Diverter}

The pumping element shown in the figure above is the Reverse Flow Diverter, RFD. The RFD operates like a three-way valve. It consists of two opposed nozzles; a symmetrical design is shown in the figure.

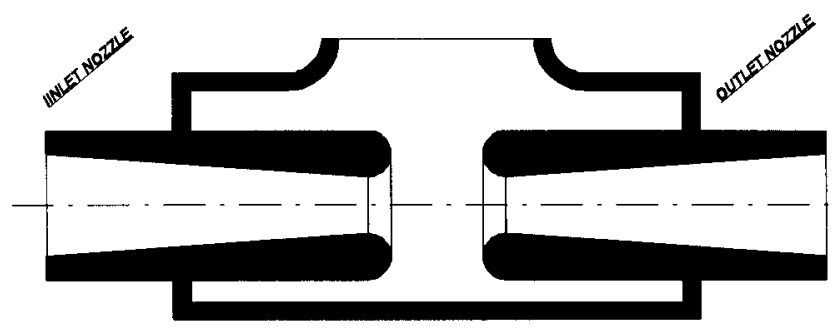

Figure A-3. Reverse Flow Diverter, RFD, Element

In operation as a pumping element, fluid enters the inlet nozzle during the drive phase, passes across the gap, entraining fluid from the supply tank, and the static pressure is then recovered along the outlet nozzle/diffuser section. Depending upon the pressure in the delivery line, fluid from the supply tank is either entrained by the flow emerging from the nozzle or, alternatively, a small proportion of the nozzle flow is fed back into the tank--so called "negative entrainment."

During the refill phase, liquid passes through the inlet nozzle (now acting as a diffuser) and into the charge vessel with only a relatively low resistance to flow produced by the well-rounded entry to the diffuser. 
HNF-3042, Revision 0

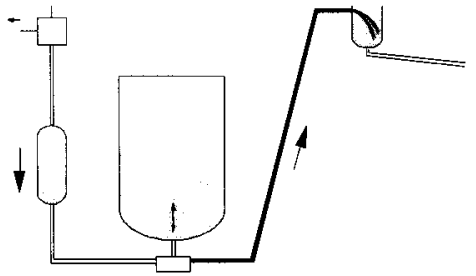

Figure A-4. Drive Phase

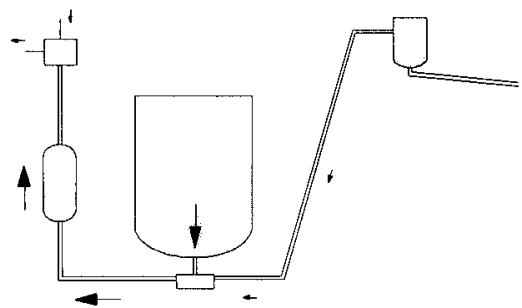

Figure A-5. Refill Phase.

\section{A.3.2 Primary Controller}

From the preceding paragraphs it can be seen that the requirements of the primary controller are to provide a positive gas flow and pressure to the charge vessel during the drive phase to allow a vent path for this gas during vent and then to provide a partial vacuum in the charge vessel during refill. This is normally accomplished using a jet pump pair which is shown schematically in Figure A-6, together with the modes of operation for the three phases of the pump's cycle.

\section{A.3.3 Secondary Controller}

The requirements of the secondary controller are to provide compressed gas to the "drive" part of the primary controller during the drive phase, to switch off the gas supply during vent, and then to supply the "suction" part of the primary controller during refill. This task is often accomplished using conventional solenoid valves which need to handle only clean gas and are 

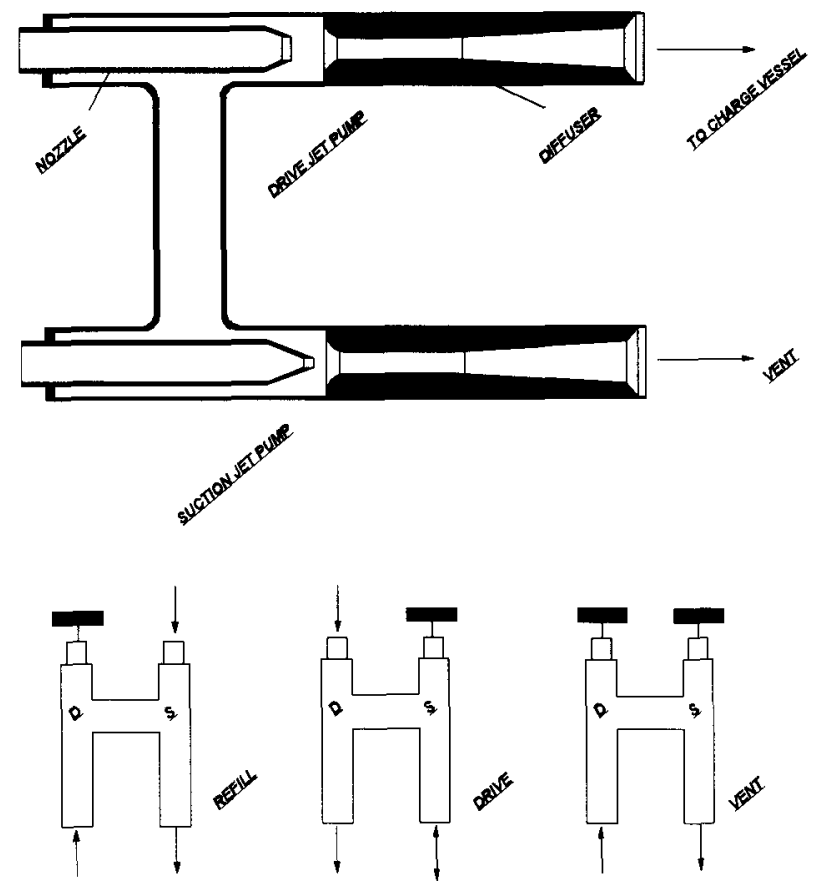

Figure A-6. Jet Pump Pair Used to Produce Drives for the Three Modes of Operation for the Three Phases of the Pump's Cycle

installed in an accessible position so that maintenance can be performed.

The other function of the secondary controller is to control the duration for which the gas is supplied to the primary controller, i.e., to set the phase times of the cycle. The method of achieving this is dependent upon the type of pumping element used as outlined below.

\section{A.3.4 Operating Cycle Limits}

During the drive (or vent) phase, if the air liquid interface passes beyond the base of the charge vessel, the compressed gas will be blown into the supply and delivery tanks which can lead to over-pressurization of these vessels and the creation of airborne aerosols. This is a 
potential fault condition known as overblow. By correct design, it is not a disastrous situation, but steps are normally taken to avoid it if possible.

At the other extreme, if an excessive vacuum is supplied to the charge vessel during the refill phase, there is the potential for sucking liquid into the primary controller and thereby discharging it into the vent system. This condition, known as flooding, is normally obviated by the correct positioning of the primary controller in relation to the charge vessel.

\section{A.3.5 Cycle Control and Stability}

The RFD pump system is normally designed with the charge vessel volume several times greater than the volume of the delivery pipe. Consequently, at the end of the refill phase, the delivery pipe has emptied (down to the level of the liquid in the supply tank), and the level of liquid in the charge vessel has risen into the air link pipe to a level compatible with the amount of vacuum supplied by the primary controller. The system is then in hydrostatic equilibrium and the liquid flow rate is zero everywhere within the pump. This condition represents a datum from which each cycle of the pump commences and is necessary to avoid cycle instability which could result in overblow.

The phase sequencing of this type of pump can therefore be achieved in an open loop manner with electronic timers, where the time of each phase of the pump is predetermined from experiment or theoretical predictions.

In a pumping system, irrespective of the type of pumping element employed, the time taken to refill the charge vessel increases as the level of liquid in the supply tank falls. The drawback to the use of electronic timers, therefore, is that in order to ensure reaching the datum position, the time for the refill phase must be set such that the charge vessel will fill even at the lowest supply tank level. This leads to very inefficient operation.

A solution to the problem is provided by the Prescon (PRESsure CONtrol) method which makes use of the observation that there is a characteristic pressure change as measured at the point $\mathrm{X}$ when the charge vessel becomes full. By detecting this pressure change, it is possible to start the drive phase immediately the charge vessel is full and so operate at optimum efficiency. This, therefore, provides a reliable, non-intrusive, closed-loop control method. In addition, the occurrence of overblow can be detected by analyzing the pressure measured at point $Y$.

\section{A.3.6 Sampling Procedure}

Each sampler comprises an RFD fluidic pump connected to a single specially designed sampling tee installed in the discharge pipework which delivers a sample of the liquid through a sample needle to the sample bottle, as indicated in Figure A-8. The flow from a fluidic pump is intermittent, i.e., there is a delivery of liquid followed by a period when the pump is refilling. This intermittent flow is used to make the sampling system operate. When the pump is delivering liquid along the delivery line, the liquid will have some velocity. As the liquid passes 
HNF-3042, Revision 0

the end of the sampling needle there is a venturi effect which draws air down the needle from a sample bottle on the other end of the needle (usually plastic with a rubber seal on the other end). As the delivery pulse from the pump ends, the liquid velocity past the needle decreases and the partial vacuum in the bottle draws liquid back into the bottle

A sample is normally collected over a number of cycles in this manner; the total amount of sample collected is governed by the length of the needle penetrating into the bottle.

Note that the sample tee design ensures that, when no sample bottle is on the needle, no liquid passes up through the needle. If a sample pump is run without a bottle, for example to purge the sample lines, the liquid flow draws a small amount of air down through the needle into the tank. Without a bottle to hold suction, there is no driving force for liquid flow up through the needle.

Where tests require it, secondary samples for comparison purposes will be taken from the sampler feed tank adjacent to the RFD using a simple 'grab' sampling technique derived from ASTM E300-92, "Standard Practice for Sampling Industrial Chemicals." 
HNF-3042, Revision 0
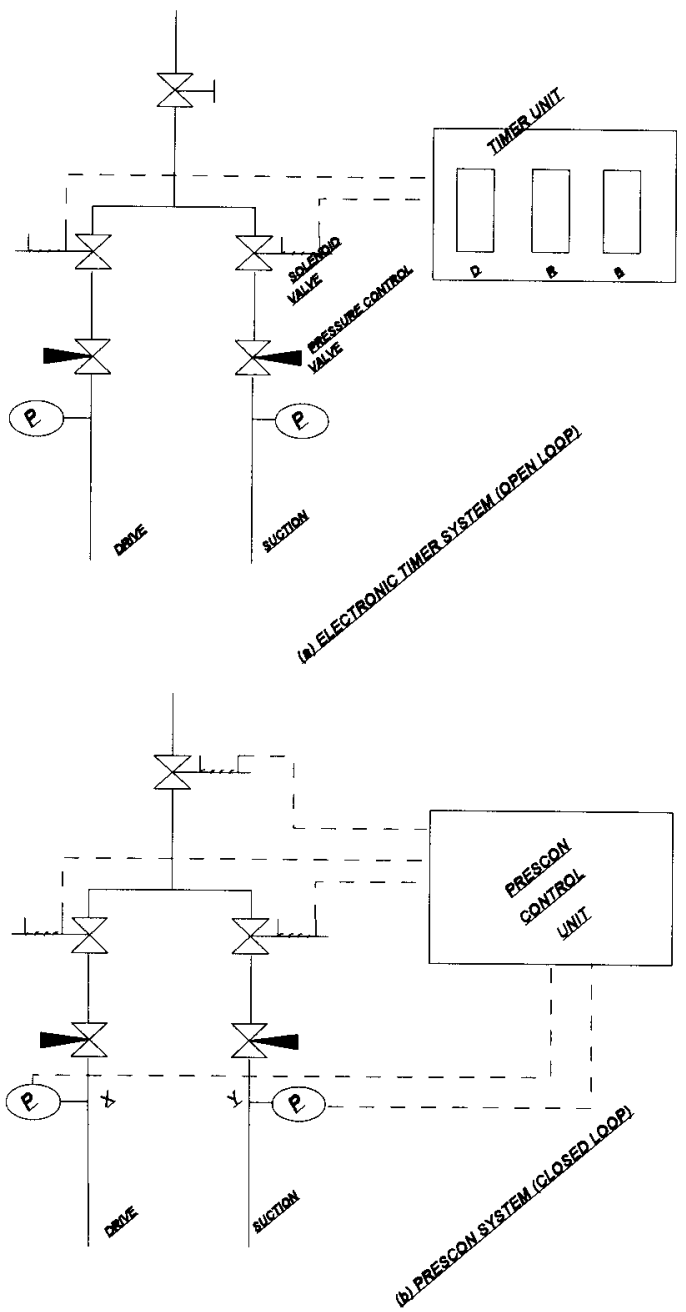

Figure A.7. Cycle Control with Timer and Pressure Control Systems. 
HNF-3042, Revision 0
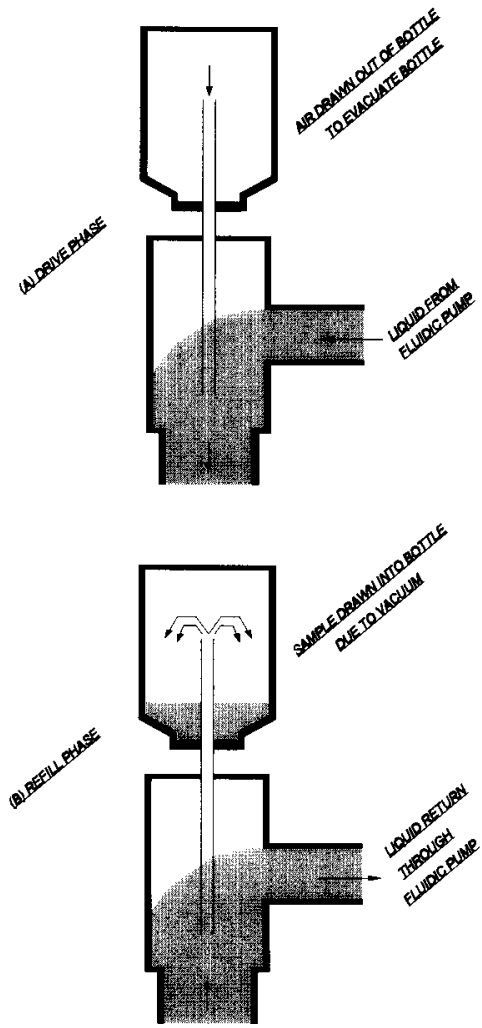

Figure A.8. Cycle Control with Timer and Pressure Control Systems. 